Ann. Sci. forest., 1974, 31 (4), 189-205.

\title{
ÉTUDE DE LA NUTRITION MINÉRALE DE PICEA EXCELSA LINK SUR SOL CALCAIRE ET SUR SOL DÉCARBONATÉ, INCIDENCE DE LA NUTRITION SUR LE MÉTABOLISME DES ANIONS MINÉRAUX ET ORGANIQUES
}

\author{
A. CLÉMENT \\ Station de Recherches sur les Sols forestiers et la Fertilisation, \\ Centre national de Recherches forestières, I.N.R.A., \\ Champenoux, 54280 Seichamps
}

\section{RÉSUMÉ}

La présente étude a été conduite sur Picea excelsa Link à partir d'analyse d'échantillons foliaires prélevés, d'une part en période de croissance printanière lorsque l'activité biologique est intense, d'autre part en hiver en période de repos biologique, sur 3 sols : un sol très carbonaté à $50 \%$ de calcaire dès la surface, un sol carbonaté à $15 \%$ de calcaire, un sol décarbonaté. Les arbres concernés sont âgés de 35 ans environ.

Les résultats obtenus mettent en évidence, sur station très calcaire, une répartition semblable des différentes combinaisons soufrées et phosphorées des tissus; les faibles teneurs en soufre et phosphore insolubles observées semblent être la conséquence d'une assimilation difficile des formes solubles respectives, assimilation qui, pour le soufre tout au moins, est liée à une synthèse protéique déficiente. Il s'établit entre l'ensemble des différentes formes azotées minérales, azotées solubles, azotées insolubles du tissu foliaire un équilibre qui ne dépend pas de la présence de calcaire dans le sol. Une des principales causes responsable de la carence en azote protéique observée sur sol calcaire serait liée à la nature de l'azote minéral disponible dans le sol.

La nutrition cationique se caractérise sur sol très calcaire notamment par une absorption importante du Ca. La majeure partie de celui-ci se trouve insolubilisée dans les tissus selon deux processus au moins, il y aurait fixation sous forme de complexes organiques insolubles non déplaçables par une résine cationique, mais aussi fixation saline sous forme d'oxalate insoluble, cette dernière se produisant surtout dans le tissu provenant de sols calcaires.

Nous avons répertorié les anions organiques essentiels qui constituent l'acidité organique libre, l'acidité organique salifiée, et mis en évidence des teneurs en acides organiques libres plus élevées dans les tissus provenant de sols carbonatés que dans ceux provenant du sol décarbonaté.

\section{INTRODUCTION}

De nombreux auteurs, dont Duchaufour (1960), Duchaufour-Bonneau (1962), Le TACON-Millier (1970), Le TACon (1972), ont montré que la présence de calcaire dans le sol avait une action directe sur la nutrition minérale d'une espèce calcifuge telle que Picea 
excelsa Link. Ces études de nutrition minérale ont été pour la plupart conduites à partir d'analyses portant sur les formes globales des éléments comme l'azote, le phosphore, le soufre, le calcium, le potassium, le magnésium. Nous avons jugé intéressant d'approfondir ces recherches en étudiant la répartition de ces éléments dans les extraits solubles et insolubles des tissus foliaires. Ceci nous a permis de mieux appréhender les problèmes de nutrition minérale en liaison avec le métabolisme des cations et des anions présents dans ces tissus.

Nous savons aujourd'hui que cations et anions sont absorbés suivant deux processus différents, il apparaît donc probable que les cations et les anions ne sont pas absorbés en quantité équivalente. Le déséquilibre électrostatique qui pəut en résulter est alors compensé par l'action régulatrice des acides organiques. Ainsi, salon Dijkhoorn (1964) on assiste lors de l'absorption d'un cation à la synthèse d'un anion organique, par contre l'absorption d'un anion minéral entraîne la décarboxylation d'un anion organique. Il est certain que la relation anions organiques-ions minéraux nous imposait d'inclure dans notre étude de nutrition minérale celles des anions organiques.

Nos observations ont été effectuées au cours de deux périodes de végétation très différentes, d'une part en période de croissance printanière, lorsque l'activité biologique est intense, d'autre part en période de repos hivernal.

\section{1. - TECHNIQUES EXPÉRIMENTALES}

\section{1. - Choix des stations}

Cette étude repose sur l'analyse foliaire d'aiguilles d'Épicéa de 3 stations forestières aux caractéristiques suivantes :

1) Une station à 50 p. 100 de calcaire dès la surface, située en forêt de Vauhalaise (Vitry-leFrançois), peuplée d'épicéas âgés de 34 ans d'une hauteur moyenne dominante de $6,40 \mathrm{~m}$. La production moyenne par ha et par an depuis l'origine à 50 ans est de $1,6 \mathrm{~m}^{3}$.

2) Une station à 15 p. 100 de calcaire dès la surface, située en forêt des Éparges (Verdun) peuplée d'épicéas, âgés de 35 ans, d'une hauteur moyenne de $11,1 \mathrm{~m}$. La production moyenne par ha et par an depuis l'origine à 50 ans est de $5 \mathrm{~m}^{3}$.

3) Une station décarbonatée, de $\mathrm{pH} 5,5$ située en forêt du Mort-Homme (Verdun) peuplée d'épicéas âgés de 37 ans, d'une hauteur moyenne de $22,8 \mathrm{~m}$. La production moyenne par ha et par an depuis l'origine à 50 ans est de $19 \mathrm{~m}^{3}$.

\section{2. - Choix des arbres : Échantillonnage}

Nous avons choisi 8 arbres dominants* par station. Les 2 prélèvements foliaires ont été effectués sur les aiguilles de l'année; le premier au printemps (début juin), en période d'intense activité biologique, le second en hiver (début janvier) en période d'activité biologique réduite.

\section{3. - Traitements des échantillons}

Les analyses ont été effectuées soit à partir du végétal vert, soit à partir du végétal séché 24 heures à $65^{\circ} \mathrm{C}$.

* Arbres dominants : ces arbres ont été sélectionnés parmi les 10 plus grands arbres de la station. 
L'analyse des formes azotées minérales et organiques ainsi que celle des acides organiques ont été conduites exclusivement sur le matériau vert. A cette fin, les échantillons prélevés sont « fixés » dès leur prélèvement sur le terrain à l'azote liquide, transportés et conservés dans des glacières portatives garnies de glace carbonique. Les échantillons ainsi congelés sont ensuite conditionnés en vue de l'extraction des différents éléments à $4{ }^{\circ} \mathrm{C}$ (Thèse CLÉMENT, 1972).

\section{4. - Analyses effectuées}

Nous avons extrait les composés anioniques et cationiques les plus labiles des tissus végétaux par deux solvants, l'eau et l'acide acétique normal, employés l'un à la suite de l'autre.

L'acide acétique normal permet d'extraire sélectivement certaines formes de $\mathrm{Ca}$ insolubles dans l'eau, ainsi que certaines combinaisons azotées également insolubles dans l'eau, tandis qu'il laisse subsister intact l'oxalate de Ca.

La différence, entre les résultats de l'analyse des éléments totaux et des éléments faisant partie des combinaisons extraites par l'eau et l'acide acétique $\mathrm{N}$, représente les éléments engagés dans des combinaisons insolubles minérales et organiques.

Chaque élément a été déterminé :

- dans sa forme soluble dans l'eau $\mathrm{P}_{\mathrm{H}_{2} \mathrm{O}}, \mathrm{S}_{\mathrm{H}_{2} \mathrm{O}}, \mathrm{NO}_{3}-\mathrm{NH}_{4}^{+}, \mathrm{N}_{\mathrm{H}_{2} \mathrm{O}}, \mathrm{Ca}_{\mathrm{H}_{2} \mathrm{O}}, \mathrm{K}_{\mathrm{H}_{2} \mathrm{O}}, \mathrm{Mg}_{\mathrm{H}_{2} \mathrm{O}}$, $\mathrm{Mn}_{\mathrm{H}_{2} \mathrm{O}}$ etc.

- dans sa forme soluble dans l'acide acétique $\mathrm{N}$ après épuisement à l'eau $\mathrm{P}_{3}, \mathrm{~S}_{\mathrm{A}}, \mathrm{N}_{\mathrm{A}}, \mathrm{Ca}_{\mathrm{A}}$, $\mathrm{K}_{\mathrm{A}}, \mathrm{Mg}_{\mathrm{A}}, \mathrm{Mn}_{\mathrm{A}}$.

- dans sa forme insoluble $P_{\mathrm{I}}, \mathrm{S}_{\mathrm{I}}, \mathrm{N}_{\mathrm{I}}, \mathrm{Ca}_{\mathrm{I}}, \mathrm{K}_{\mathrm{I}}, \mathrm{Mg}_{\mathrm{I}}, \mathrm{Mn}_{\mathrm{I}}$,

Nous avons complété cette recherche par l'étude globale des anions organiques présents sous forme d'acides libres dans les tissus (acidité organique libre*), et par la détermination globale des anions organiques salifiés (acidité organique salifiée). Nous avons également procédé à l'évaluation de l'acidité minérale libre (acides minéraux) et à l'évaluation de l'acidité minérale salifiée.

Pour ce faire, nous avons déterminé après déplacement des cations par une résine cationique et dosage jusqu'à $\mathrm{pH}=8$ l'acidité totale, c'est-à-dire la somme des anions liés soit à un proton, soit à un cation métallique (acidité libre + acidité salifiée). Nous avons également mesuré l'acidité totale libre (anions liés à un proton) par neutralisation à $\mathrm{pH}=8$ de la solution d'extraction du tissu végétal.

Enfin, à partir du pH des extraits foliaires, des quantités de soufre et de phosphore solubles présentes dans les tissus, nous avons estimé l'acidité minérale totale. Cette acidité est composée de l'acidité minérale libre (acides minéraux, sulfates acides et phosphates acides) et de l'acidité minérale salifiée (sels minéraux, sulfates acides et phosphates acides).

A partir des données précédentes, il nous a été ainsi possible de calculer l'acidité organique libre et salifiée des tissus foliaires.

Nous avons tenu à préciser également la nature des acides organiques et de leurs sels, leur identification a été effectuée par chromatographie sur papier Wattman en simple et double dimension.

Solvants de migration utilisés :

solvant $\mathrm{n}^{\circ} 1$ : Éthylméthylcétone/acétone/eau/acide formique,

solvant $\mathrm{n}^{\circ} 2: \mathrm{NH}_{4} \mathrm{OH} /$ alcool éthylique/eau,

solvant no $3:$ n Butanol/acide acétique/eau.

En chromatographie monodimensionnelle, le développement a lieu par voie descendante avec le solvant $\mathrm{n}^{0} 1$.

En chromatographie bidimensionnelle, le développement a lieu par voie descendante en utilisant dans l'ordre les solvants 2 et 3 pour les première et deuxième dimensions.

Les chromatogrammes sont révélés au bleu de bromophénol et au sulfate de cuivre. L'estimation quantitative des taches est obtenue par photodensitométrie d'après les techniques proposées par S. BRUCKERT (1970).

* Représente une acidité potentielle à $\mathrm{pH}=8$. Sont donc essentiellement concernés les acides organiques libres dont le pk est égal ou inférieur à 8 . 


\section{5. - Méthodes statistiques d'interprétation}

Les résultats exposés aux tableaux 1 et 2 correspondent chacun à une moyenne obtenue à partir de 8 mesures séparées effectuées sur chaque station. La différenciation des moyennes entre elles s'appuie sur une analyse de la variance à 1 facteur contrôlé. Le facteur contrôlé est le facteur station.

\section{2. - RÉSULTATS ET DISCUSSION}

\section{1. - AsPeCT GÉNÉRAL}

Les résultats obtenus au cours des deux périodes de prélèvement diffèrent fondamentalement. Ceci semblait prévisible puisque notre échantillonnage est effectué à deux époques différentes, correspondant pour la $1^{\text {re }}$ à une période d'activité biologique intense (prélèvement le printemps, tableau 1), pour la seconde à une période d'activité biologique réduite (prélèvement d'hiver, tableau 2). Des observations analogues portant sur d'autres espèces résineuses ont déjà été signalées par de nombreux auteurs. Relevons TAMM (1955), WeLLS et Metz (1963). Le prélèvement printanier se caractérise par des aiguilles dont le poids correspond en moyenne à 50 p. 100 de leur poids de fin de végétation. Le pH des tissus apparaît très acide, notamment pour la période de croissance printanière $: 3$ sur station très carbonatée, 3,2 sur station décarbonatée. Nous reviendrons ultérieurement sur la signification de ces résultats qui nous permettent déjà une estimation globale de l'équilibre physiologique de l'arbre vis-à-vis du milieu sol. Les différentes formes de soufre, le Ca insoluble et le Ca soluble dans l'eau augmentent en teneur lorsque le tissu foliaire évolue du stade juvénile au stade adulte. Inversement, les autres éléments ou combinaisons analysés présentent des teneurs constantes ou décroissantes au cours du vieillissement des tissus.

Notons que les chiffres du tableau 3 ont été obtenus à partir d'un seul échantillon choisi comme le plus représentatif de la teneur moyenne stationnelle en anions organiques. On ne s'étonnera donc pas que certains chiffres qui devraient être égaux dans les tableaux 2 et 3 soient légèrement différents. Par exemple, l'acidité minérale totale du tableau 3 et la somme des acidités minérales libres et salifiées du tableau 2.

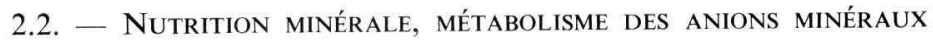

\section{1. - Nutrition anionique}

\section{1. - Nutrition en phosphore.}

Au stade de croissance printanier, les teneurs en phosphore total $\left(\mathbf{P}_{\mathrm{T}}\right)$ des tissus ne dépendent pas de la présence de calcaire dans le sol, alors que a priori, on pouvait s'attendre à une carence de cet élément dès le stade de croissance juvénile de l'aiguille sur sol calcaire. Ce n'est qu'au cours du prélèvement hivernal que se manifeste le phénomène de carence bien connu par les spécialistes de nutrition minérale. Au printemps, un sol très riche en calcaire favorise l'accroissement des teneurs des tissus foliaires en combinaisons solubles dans l'eau $\left(\mathrm{P}_{\mathrm{H}_{2} \mathrm{O}}\right)$. Par contre, la quantité de phosphore insolubilisée sous forme de composés 
TABLEAU 1 - TABLE 1

Concentrations moyennes des anions et cations des prélèvements foliaires effectués en période printanière sur Épicéa

Les résultats sont exprimés d'une part en mé/100 $\mathrm{g}$ de végétal sec pour les cations sauf $\mathrm{NH}_{4}{ }^{+}$et pour les acidités organiques et minérales, d'autre part, en mg pour les anions minéraux et éléments organisés.

Medium contents of anions and cations on foliar sample of Spruce during the spring time The results are expressed in me/100 $\mathrm{g}$ of dry matter for cations except $\mathrm{NH}_{4}{ }^{+}$and for organic and mineral acidity, in $\mathrm{mg}$ for mineral anions and others combinations.

\begin{tabular}{|c|c|c|c|c|c|c|}
\hline \multirow[b]{2}{*}{$\begin{array}{c}\text { Symbole } \\
\text { d'identification }\end{array}$} & \multicolumn{3}{|c|}{$\begin{array}{l}\text { Résultats correspondant à la moyenne } \\
\text { des } 8 \text { arbres pris par station }\end{array}$} & \multicolumn{3}{|c|}{$\begin{array}{l}\text { Signification entre } \\
\text { station par le test de FISCHER }\end{array}$} \\
\hline & $\begin{array}{c}\text { Station } \\
\text { très } \\
\text { carbonatée } \\
\text { m } 1\end{array}$ & $\begin{array}{c}\text { Station } \\
\text { carbonatée } \\
\text { m2 }\end{array}$ & $\begin{array}{c}\text { Station } \\
\text { décarbonatée } \\
\text { m3 }\end{array}$ & $\begin{array}{l}\text { Station } \\
(1-2)\end{array}$ & $\begin{array}{c}\text { Station } \\
(2-3)\end{array}$ & $\begin{array}{c}\text { Station } \\
(1-3)\end{array}$ \\
\hline 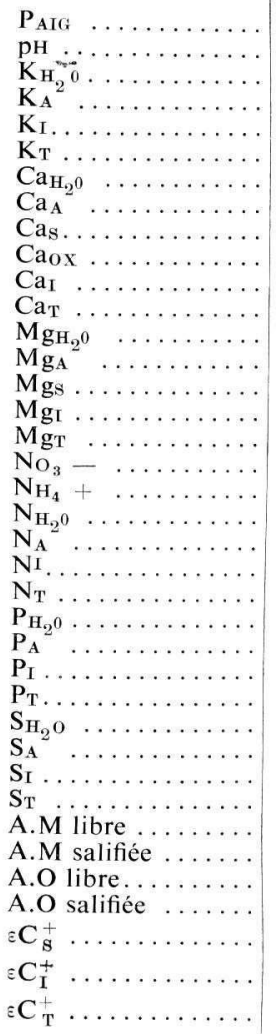 & $\begin{array}{c}0,200 \\
3,0 \\
26,1 \\
0,2 \\
\text { trace } \\
23,2 \\
1,1 \\
3,3 \\
4,5 \\
3,0 \\
21,5 \\
26,5 \\
6,7 \\
0,3 \\
7,0 \\
0,1 \\
7,1 \\
4,7 \\
3,7 \\
81,5 \\
8,8 \\
955 \\
1054 \\
129,8 \\
5,8 \\
88,3 \\
224,2 \\
37,4 \\
\text { trace } \\
49,1 \\
86,6 \\
4,3 \\
6,7 \\
76,7 \\
35,5 \\
37,9 \\
18,9 \\
56,8 \\
5\end{array}$ & $\begin{array}{c}0,268 \\
3,0 \\
27,5 \\
0,4 \\
\text { trace } \\
23,9 \\
1,0 \\
4,0 \\
5,1 \\
0,2 \\
18,6 \\
23,1 \\
6,2 \\
0,3 \\
6,6 \\
1,0 \\
7,5 \\
4,8 \\
4,6 \\
76,2 \\
22,0 \\
117 \\
1325 \\
105,6 \\
5,8 \\
107,5 \\
218,9 \\
33,2 \\
\text { trace } \\
71,8 \\
105,1 \\
3,6 \\
5,6 \\
75,9 \\
35,2 \\
39,7 \\
15,5 \\
55,3 \\
32\end{array}$ & $\begin{array}{c}0,269 \\
3,20 \\
23,7 \\
0,3 \\
\text { trace } \\
23,3 \\
0,8 \\
3,4 \\
4,2 \\
3,3 \\
16,2 \\
20,5 \\
5,1 \\
0,4 \\
5,5 \\
1,6 \\
7,1 \\
4,0 \\
8,8 \\
108,2 \\
33,1 \\
135 \\
1540 \\
97,9 \\
8,1 \\
123,1 \\
229,2 \\
20,0 \\
\text { trace } \\
81,1 \\
101,3 \\
3,4 \\
4,7 \\
57,3 \\
32,8 \\
33,8 \\
17,1 \\
51,0 \\
35\end{array}$ & $\begin{array}{l}\mathrm{XX} \\
\mathrm{XX} \\
\mathrm{XX} \\
\mathrm{X}\end{array}$ & $\begin{array}{l}\text { XX } \\
X X \\
X X \\
X X \\
X X \\
X X\end{array}$ & $\begin{array}{l}\text { XX } \\
\text { XX } \\
\text { XX } \\
\text { XX } \\
\text { X } \\
\text { XX } \\
\text { XX } \\
\\
\text { XX } \\
\text { XX } \\
\text { XX } \\
\text { XX } \\
\text { XX } \\
\text { XX } \\
\text { XX } \\
\text { XX } \\
\text { XX } \\
\text { XX } \\
\text { X } \\
\text { XX } \\
\text { XX } \\
\text { XX } \\
\text { XX }\end{array}$ \\
\hline
\end{tabular}

* Signification au risque de $5 \%$. ** Signification au risque de $1 \%$.

N.B. Les indices $\mathrm{H}_{2} \mathrm{O}, \mathrm{A}, \mathrm{S}, \mathrm{I}, \mathrm{T}$, signifient que l'élément concerné est pris dans sa forme soluble dans l'eau, soluble dans l'acide acétique $\mathrm{N}$ après épuisement à l'eau, soluble globalement dans les 2 formes précédentes, insoluble, ou totale.

$\mathrm{P}_{\mathrm{AIG}}$ correspond au poids de 100 aiguilles en $\mathrm{g}$.

$\varepsilon \mathrm{C}^{+}$représente la somme des cations. A.M représente l'acidité minérale. A.O représente l'acidité organique.

$\mathrm{K}_{\mathrm{H}_{2} \mathrm{O}}$ peut être supérieur à $\mathrm{K}_{\mathrm{T}}$ par le fait que le $\mathrm{K}$ est quasiment sous forme soluble, et que les analyses ont été effectuées sur des prises d'essais différentes. 


\section{TABLEAU 2 - TABLE 2}

\section{Concentrations moyennes des anions et cations des prélèvements foliaires effectués} en période hivernale sur Épicéa

Les résultats sont exprimés d'une part en mé/100 $\mathrm{g}$ de végétal sec pour les cations sauf $\mathrm{NH}_{4}^{+}$et pour les acidités organiques et minérales, d'autre part en mg pour les anions minéraux et éléments organisés.

Medium contents of anions and cations on foliar sample of Spruce during the winter time The results are expressed in me/100 $\mathrm{g}$ of dry matter for cations exceptNH ${ }_{4}^{+}$and for organic and mineral acidity, in $\mathrm{mg}$ for mineral anions and others combinations.

\begin{tabular}{|c|c|c|c|c|c|c|}
\hline \multirow{2}{*}{$\begin{array}{c}\text { Symbole } \\
\text { d'identification }\end{array}$} & \multicolumn{3}{|c|}{$\begin{array}{l}\text { Résultats correspondant à la moyenne } \\
\text { des } 8 \text { mesures effectuées par station }\end{array}$} & \multicolumn{3}{|c|}{$\begin{array}{l}\text { Signification entre station } \\
\text { par le test de FISCHER }\end{array}$} \\
\hline & $\begin{array}{c}\text { Station } \\
\text { très } \\
\text { carbonatée } \\
\text { m } 1\end{array}$ & $\begin{array}{c}\text { Station } \\
\text { carbonatée } \\
\mathrm{m} 2\end{array}$ & $\begin{array}{c}\text { Station } \\
\text { décarbonatée } \\
\mathrm{m} 3\end{array}$ & $\begin{array}{l}\text { Station } \\
(1-2)\end{array}$ & $\begin{array}{l}\text { Station } \\
(2-3)\end{array}$ & $\begin{array}{l}\text { Station } \\
(1-3)\end{array}$ \\
\hline 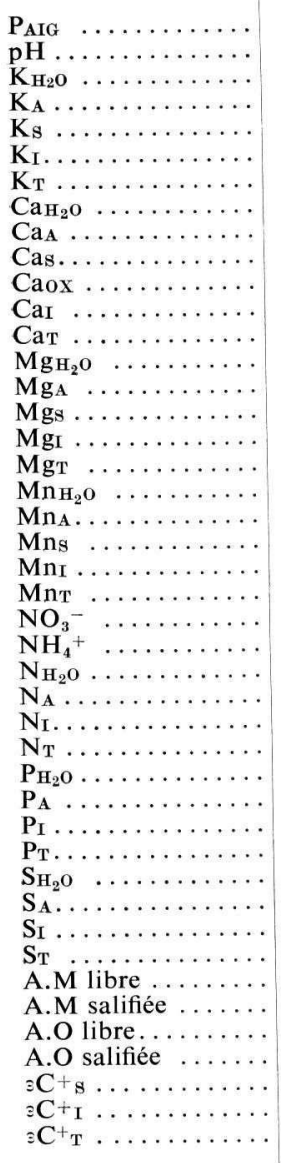 & $\begin{array}{c}0,474 \\
3,7 \\
14,1 \\
0,5 \\
14,6 \\
\text { trace } \\
15,1 \\
4,4 \\
2,4 \\
6,9 \\
8,8 \\
41,5 \\
48,4 \\
5,6 \\
1,0 \\
6,7 \\
0,2 \\
7,0 \\
0,1 \\
\text { trace } \\
0,1 \\
\text { trace } \\
0,1 \\
1,8 \\
2,0 \\
30,2 \\
4,8 \\
750 \\
788 \\
71,3 \\
4,1 \\
66,6 \\
142,0 \\
42,9 \\
\text { trace } \\
54,8 \\
97,8 \\
2,4 \\
5,9 \\
34,8 \\
32,7 \\
28,4 \\
42,3 \\
70,8\end{array}$ & $\begin{array}{c}0,488 \\
3,7 \\
19,2 \\
0,7 \\
20,0 \\
\text { trace } \\
18,7 \\
3,9 \\
2,8 \\
6,8 \\
0,4 \\
25,1 \\
32,0 \\
5,0 \\
1,1 \\
6,2 \\
0,3 \\
6,6 \\
\text { trace } \\
\text { trace } \\
\text { trace } \\
\text { trace } \\
\text { trace } \\
0,2 \\
2,2 \\
31,6 \\
8,4 \\
1054 \\
1097 \\
59,5 \\
6,0 \\
88,5 \\
153,8 \\
51,5 \\
\text { trace } \\
76,6 \\
128,1 \\
2,3 \\
4,9 \\
35,4 \\
28,7 \\
33,1 \\
24,2 \\
57,4 \\
09\end{array}$ & $\begin{array}{c}0,473 \\
3,9 \\
19,0 \\
0,8 \\
19,9 \\
\text { trace } \\
18,8 \\
2,4 \\
3,0 \\
5,5 \\
1,3 \\
34,8 \\
40,3 \\
4,7 \\
1,4 \\
6,2 \\
0,4 \\
6,6 \\
1,9 \\
0,6 \\
2,6 \\
\text { trace } \\
2,2 \\
0,8 \\
4,3 \\
39,6 \\
12,1 \\
1254 \\
1311 \\
106,7 \\
6,3 \\
82,8 \\
196,1 \\
31,0 \\
\text { trace } \\
87,2 \\
118,5 \\
3,6 \\
5,5 \\
25,9 \\
29,5 \\
34,3 \\
33,7 \\
68,0 \\
34\end{array}$ & $\begin{array}{l}X X \\
X X \\
X X \\
X X \\
X X\end{array}$ & $\begin{array}{l}\text { XX } \\
\text { XX } \\
X X \\
X X \\
X \\
X X \\
X X \\
X X \\
\text { XX } \\
\text { XX }\end{array}$ & $\begin{array}{l}\text { XX } \\
X X \\
X X \\
X X \\
X X \\
\text { XX } \\
\text { XX } \\
\text { XX } \\
\text { XX } \\
\text { XX } \\
\text { XX } \\
\text { X } \\
\text { XX } \\
\text { XX }\end{array}$ \\
\hline
\end{tabular}

N.B. - $\mathrm{Mn}_{\mathrm{H}_{2} \mathrm{O}}$ peut être supérieur à $\mathrm{Mn}_{\mathrm{T}}$ par le fait que le $\mathrm{Mn}$ est quasiment sous forme soluble et que les analyses ont été effectuées sur des prises d'essais différentes.

- Il en est de même pour $\mathrm{K}$. 
TABLEAU 3 - TABLE 3

Identification des principaux anions organiques du tissu foliaire d'Épicéa réalisée sur des prélèvements hivernaux

Les résultats sont exprimés en mé/100 $\mathrm{g}$ de végétal sec. Ces anions sont représentés par la somme des anions organiques liés à un proton (acides organiques libres), et des anions organiques salifiés présents dans les tissus.

Identification of principal organic anions in Spruce foliar realized on winter samples

The results are expressed in me/100 $\mathrm{g}$ of dry matter. These anions are composed of the organic anions fastened to $\mathrm{H}^{+}$(organic acids) and organic anions fastened to metals (organic salts) in the foliar tissues.

\begin{tabular}{|c|c|c|c|c|c|c|c|}
\hline & \multicolumn{2}{|l|}{ Stations } & \multicolumn{2}{|c|}{$\begin{array}{l}\text { Anions } \\
\text { totaux }\end{array}$} & \multicolumn{2}{|c|}{$\begin{array}{l}\text { Anions } \\
\text { minéraux }\end{array}$} & $\begin{array}{c}\text { Anions } \\
\text { organiques } \\
\text { totaux } \\
\text { (1) }\end{array}$ \\
\hline \multicolumn{3}{|c|}{$\begin{array}{l}\text { Station très carbonatée } \ldots \ldots \ldots \ldots \\
\text { Station décarbonatée } \ldots \ldots \ldots \ldots\end{array}$} & \multicolumn{2}{|c|}{$\begin{array}{l}76 \\
68\end{array}$} & \multicolumn{2}{|c|}{6} & $\begin{array}{l}70 \\
59\end{array}$ \\
\hline \multicolumn{6}{|c|}{ Anions aliphatiques et alicycliques } & \multirow{2}{*}{$\begin{array}{l}\text { Total } \\
\text { (2) }\end{array}$} & \multirow{2}{*}{$\begin{array}{c}\text { Indosés } \\
\text { (1)-(2) }\end{array}$} \\
\hline Am* & A.s & A.c & A.q & A.sh & A.Ox & & \\
\hline 4 & traces & 2 & 19 & 24 & 16 & 65 & 5 \\
\hline 4 & traces & 4 & 8 & 27 & 9 & 52 & 7 \\
\hline
\end{tabular}

* A.m correspond à Anion malique

A.s correspond à Anion succinique

A.c correspond à Anion citrique

A.q correspond à Anion quinique

A.sh correspond à Anion shikimique

A.ox correspond à Anion oxalique

N.B. - Les anions totaux correspondent à l'acidité libre augmentée de l'acidité de déplacement des cations par une résine échangeuse de protons.

organiques $\left(\mathrm{P}_{\mathrm{I}}\right)$ apparaît moins importante que celle observée lorsqu'il n'y a pas de calcaire dans le sol. Le rapport $\frac{P_{\mathrm{H}_{2} \mathrm{O}}}{\mathrm{P}_{\mathrm{I}}}$ confirme les observations précédentes puisqu'il est de 1,47 sur station très carbonatée et de 0,80 sur station décarbonatée.

L'abondance des formes solubles dans l'eau ne semble pas être la conséquence d'une absorption intense du phosphore minéral du sol, mais apparaît plutôt être le résultat d'une organisation déficiente de celui-ci par le végétal. Il y aurait sur sol très calcaire accumulation des formes solubles $\mathrm{PO}_{4} \mathrm{H}_{2}{ }^{-}$dans les tissus foliaires par défaut d'assimilation de l'anion minéral.

Notons enfin que les teneurs en $\left(\mathrm{P}_{\mathrm{H}_{2} \mathrm{O}}\right)$ observées au stade de croissance printanier régressent en fin de cycle de végétation, cette régression s'opérant de telle sorte que les teneurs apparaissent pour les 3 stations, statistiquement identiques au stade du prélèvement hivernal (tableaux 1 et 2 ). 


\section{2. - Nutrition en soufre.}

A la différence du phosphore, la teneur en soufre total des tissus $\left(\mathrm{S}_{\mathrm{T}}\right)$ est dès le printemps significativement plus forte dans le sol décarbonaté que dans le sol très carbonaté.

La différenciation entre station très calcaire, calcaire et décarbonatée est plus marquée encore si l'on considère les combinaisons insolubles et solubles dans l'eau $\left(\mathrm{S}_{\mathrm{I}}\right.$ et $\left.\mathrm{S}_{\mathrm{H}_{2} \mathrm{O}}\right)$. Il apparaît notamment que 15 p. 100 de calcaire dans le sol (station carbonatée) suffit à modifier très significativement les teneurs en $\mathrm{S}_{\mathrm{H}_{2} \mathrm{O}}$ et $\mathrm{S}_{\mathrm{I}}$ du tissu foliaire. On observe en effet, pour chaque période de prélèvement, que la distribution des formes $\mathrm{S}_{\mathrm{H}_{2} \mathrm{O}}$ et $\mathrm{S}_{\mathrm{I}}$ s'inverse selon la présence ou l'absence de calcaire.

Il ne semble pas que l'abondance des formes solubles $\mathrm{S}_{\mathrm{H}_{2} \mathrm{O}}$ soit la conséquence de l'absorption intense du soufre minéral du sol mais plutôt, comme pour le phosphore, le résultat d'une organisation déficiente du soufre minéral $\left(\mathrm{SO}_{4}{ }^{-}\right)$sur sol calcaire. Remarquons cependant qu'à l'opposé du phosphore, la teneur dans les tissus foliaires s'accroît au cours de l'année de telle sorte qu'en fin de végétation, elle apparaît beaucoup plus importante qu'au stade de croissance juvénile.

\section{3. - Nutrition azotée.}

Il est prouvé que le calcaire a une influence très importante sur le bilan azoté de l'Épicéa; l'analyse des différentes formes azotées présentes dans les tissus foliaires nous a permis de la préciser.

\section{Azote minéral.}

Il est apparu, en période ou non d'activité biologique, que la teneur en azote minéral sous forme nitrique du tissu foliaire est très faible et ne diffère pas suivant les stations. A l'inverse, l'azote ammoniacal $\left(\mathrm{NH}_{4}{ }^{+}\right)$est toujours plus abondant dans la station décarbonatée que dans les deux autres. Les faibles teneurs en $\mathrm{NH}_{4}{ }^{+}$et $\mathrm{NO}_{3}{ }^{-}$enregistrées montrent que l'accumulation puis la réduction des nitrates, l'accumulation de l'ammonium puis sa transformation en acide aminé, ne se produisent pas au niveau des tissus foliaires mais plutôt au niveau des racines. C'est d'ailleurs ce que note Heller dans son traité de physiologie végétale (1969).

Azote organique soluble dans l'eau $\left(\mathrm{N}_{\mathrm{H}_{2} \mathrm{O}}\right)$ et soluble dans l'acide acétique $\mathrm{N}$ après épuisement à l'eau $\left(\mathrm{N}_{\mathrm{A}}\right)$.

Ces combinaisons caractérisent notamment les acides aminés et les amides, et pour la forme NA les peptides et les protéines solubles, mais cette liste est loin d'être exhaustive.

En période d'activité biologique intense, comme en période d'activité biologique réduite, ces composés azotés reflètent, pour les conditions de sols choisies, l'état de nutrition azotée de l'Épicéa : les deux formes de l'azote augmentent régulièrement lorsque la teneur du sol en calcaire diminue. Peuvent-ils constituer un nouveau critère servant à définir les conditions de nutrition azotée? Il est difficile, en ce qui nous concerne, de l'affirmer pour le moment, une 
étude complémentaire étant nécessaire. Notons cependant que, dans un même ordre d'idée, Barnes et Bengton (1968) ont montré que la détermination au niveau des rameaux d'un acide aminé tel que l'arginine pouvait servir d'indicateur de nutrition azotée.

\section{Azote insoluble $(\mathrm{N})_{1}$.}

Il constitue la fraction azotée la plus importante des tissus foliaires, celle-ci représente environ $90 \%$ de l'azote total contenu dans l'aiguille. Il a été maintes fois observé que la présence de calcaire dans le sol agissait sur la teneur en azote total des tissus foliaires au point de carencer l'Épicéa. Ce fait confirme que l'azote insoluble peut servir, au même titre que l'azote total, de diagnostic pour estimer l'état de nutrition azotée.

\section{Relations entre les diverses formes azotées des tissus foliaires.}

L'étude des rapports azote nitrique sur azote ammoniacal, azote minéral sur azote soluble, azote soluble sur azote insoluble, permet d'émettre quelques hypothèses concernant la nutrition, l'assimilation et le métabolisme de l'azote chez l'Épicéa sur sol très carbonaté et décarbonaté.

\section{Rapport $\mathrm{NO}_{3}-/ \mathrm{NH}_{4}{ }^{+}$.}

Les travaux de LE TACON (1972) ont montré que dans les sols calcaires, l'azote minéral disponible pour le végétal était essentiellement représenté par de l'azote sous forme nitrique, alors que en sol non calcaire la forme ammoniacale était dominante.

L'azote minéral présent dans les tissus foliaires pourrait refléter, malgré sa faible teneur par rapport à l'azote protéique, le type d'azote minéral disponible dans le sol pour le végétal. Il apparaît en effet, si on caractérise l'azote minéral des tissus foliaires par le rapport $\mathrm{NO}_{3}-1$ $\mathrm{NH}^{+}{ }_{4}$, que le végétal subit en sol très carbonaté une nutrition à dominance nitrique alors que en sol décarbonaté cette nutrition est essentiellement ammoniacale. Ce rapport passe de 1,3 sur station très carbonatée à 0,45 sur station décarbonatée.

\section{Rapport azote minéral/azote soluble*}

Ce rapport est indépendant de la station étudiée, il est voisin de 0,09.

\section{Rapport azote soluble/azote insoluble.}

Ce rapport est indépendant de la station étudiée et voisin de 0,09 comme le précédent.

Ces résultats montrent que, dans le tissu foliaire d'Épicéa, il s'établit entre l'ensemble des formes azotées minérales, des formes azotées solubles et insolubles un équilibre ne dépendant pas de la présence de calcaire du sol. De ce fait, on peut penser qu'une des principales causes responsables de la carence en azote insoluble observée sur sol calcaire doit être liée à la nature de l'azote minéral du sol et non au blocage du métabolisme azoté au niveau d'une forme azotée organique soluble.

* L'azote soluble comprend les formes solubles dans l'eau et dans l'acide acétique N après épuisement à l'eau. 
Si l'absorption puis l'assimilation du cation $\mathrm{NH}_{4}{ }^{+}$provenant du sol ne pose pas a priori de difficultés, il ne semble pas qu'il en soit de même pour l'anion $\mathrm{NO}_{3}^{-}$; ce dernier en effet, pour passer après réduction de $\mathrm{NH}_{3}$ à $\mathrm{NH}_{4}{ }^{+}$, nécessite la synthèse d'un acide organique (DIJKSHOORN, 1964).

Les teneurs élevées en acides organiques observées dans le tissu foliaire provenant des sols calcaires apparaissent en liaison directe avec la nutrition nitrique, si l'on admet que le végétal est contraint de synthétiser certains acides organiques pour assurer le passage " nitrate réduit sous forme $\mathrm{NH}_{3}$ " en dérivé aminé. Ces acides ne semblent cependant que très partiellement utilisés pour la synthèse de ces dérivés aminés, la raison de cette salification très partielle pourrait être liée à une faible disponibilité des formes $\mathrm{NH}_{3}$ provenant de la réduction des nitrates. Les fortes teneurs en acides organiques dépendraient donc de l'activité des enzymes nitrate et nitrite réductases. Ce que nous avons observé dans une autre étude portant sur l'activité de la nitrate réductase (CLÉMENT-LE TACON, sous presse, 1975) confirme notre hypothèse : nous avons en effet mis en évidence sur sol calcaire, une activité moins importante de la nitrate réductase dans les racines d'Épicéa que dans les racines de Pin noir.

Notons enfin que Hoffmann F. et Fiedler H.-J. (1966) ont relevé chez de jeunes résineux une difficulté d'absorption des nitrates.

\section{4. - Relations entre les nutritions en azote et en soufre.}

DIJKhOORN et VAN WIJK (1967) ont montré qu'il existait un rapport S organique/N organique propre à chaque espèce végétale, indépendant du sol sur lequel s'élabore le tissu foliaire; la valeur de ce rapport apparaît étroitement liée à la synthèse protéique. Ces observations ont été confirmées par Kelly et Lambert (1972). Pour Pinus radiata, ces derniers montrent notamment que le rapport est indépendant du sol mais aussi de la période du prélèvement foliaire.

Nous avons retrouvé chez Picea excelsa Link cette indépendance vis-à-vis du sol tout au moins en ce qui concerne le prélèvement hivernal. Ce rapport demeure voisin de celui de Pinus radiata $(0,068)$, il est en effet de 0,067 sur station décarbonatée et de 0,070 sur station carbonatée et très carbonatée. Ceux-ci sont quelque peu différents en période d'activité biologique intense (prélèvement de printemps) et ont pour valeurs respectives : 0,053, 0,055, 0,047 lorsque l'on passe de la station décarbonatée à la station très carbonatée.

La comparaison du rapport $\mathrm{S}$ organique/ $\mathrm{N}$ organique au rapport $\mathrm{S}$ total/ $\mathrm{N}$ total permet de mettre en évidence des valeurs de $\mathrm{S}$ total/ $\mathrm{N}$ total supérieures aux valeurs de $\mathrm{S}$ organique/ $\mathrm{N}$ organique. Si l'on souscrit à l'interprétation donnée par KELLY et LAMBERT, il apparaît que la carence azotée observée sur station calcaire n'est pas la conséquence d'une carence en soufre, celui-ci ayant au contraire tendance à s'accumuler sous forme soluble par manque d'absorption d'azote. Notons que nous n'avons pas relevé de relations similaires entre la nutrition azotée et la nutrition en phosphore. 


\section{2. - Nutrition cationique}

Les analyses correspondant aux deux périodes de prélèvement nous montrent qu'au cours du vieillissement, la teneur globale en cations bivalents s'accroît tandis que décroît la teneur en monovalents.

\section{1. - Potassium.}

$\mathrm{K}$ se trouve dans le tissu foliaire en quasi-totalité sous forme labile, donc sous forme de sels minéraux et organiques solubles. Nous n'avons pas relevé une influence nette du calcaire sur les teneurs en $\mathrm{K}$ du tissu foliaire.

\section{2. - Magnésium.}

La plus grande partie du $\mathrm{Mg}$ (entre 80 et $99 \%$ suivant le prélèvement et la station) se trouve dans le tissu foliaire sous forme soluble. Nous avons observé des teneurs identiques en $\mathrm{Mg}$ total aussi bien en sol carbonaté qu'en sol décarbonaté, en période ou non d'activité biologique. Cette valeur constante du $\mathrm{Mg}_{\mathrm{T}}$ cache au printemps une concentration en $\mathrm{Mg}$ soluble dans l'eau $\left(\mathrm{Mg}_{\mathrm{H}_{2} \mathrm{O}}\right)$ plus grande dans les tissus provenant des stations calcaires; par contre, une concentration en $\mathrm{Mg}$ insoluble $\left(\mathrm{Mg}_{\mathrm{I}}\right)$ plus faible.

\section{3. - Calcium.}

La teneur en calcaire du sol agit sur la teneur en Ca du tissu foliaire. Le Ca absorbé en quantité importante par l'Épicéa, sur sol calcaire, semble rapidement éliminé des sucs tissulaires par insolubilisation. Ce processus d'insolubilisation se manifeste déjà dans le tissu foliaire juvénile. A cette époque, la teneur moyenne en $\mathrm{Ca}_{\mathrm{I}}$ s'établit à 50 p. 100 environ de la teneur moyenne observée en fin de cycle de végétation. Notons aussi que les teneurs en Ca soluble dans l'eau $\left(\mathrm{Ca}_{\mathrm{H}_{2} \mathrm{O}}\right)$ différencient les stations carbonatées de la station décarbonatée et ce aussi bien en période d'activité biologique qu'en période d'inactivité. Il est également curieux de constater qu'indépendamment de la station, les teneurs en $\left(\mathrm{Ca}_{\mathrm{H}_{2} \mathrm{O}}\right)$ relevées en fin de cycle de végétation sont de 3 à 4 fois plus élevées qu'en période de croissance printanière où l'activité biologique est intense. Remarquons qu'à l'inverse, la concentration en $\mathrm{Ca}_{\mathrm{A}}$ baisse lorsque le tissu vieillit.

\section{4. - Bilan cationique.}

En période d'activité biologique, il se produit sur stations calcaires une accumulation des cations solubles plus importante que sur station décarbonatée; alors qu'on observe en période d'inactivité biologique le phénomène inverse. Remarquons également que les teneurs en insolubles $\mathrm{sC}_{\mathrm{I}}^{+}$sur les trois stations étudiées en période de croissance printanière ne sont pas significativement différentes.

\section{3. - INCIDENCE DE LA NUTRITION MINÉRALE SUR LA SYNTHÈSE OU LA DÉGRADATION DES ANIONS ORGANIQUES DES TISSUS}

23.1. - Il est prouvé que l'absorption minérale conditionne très fortement la synthèse ou la biodégradation des anions organiques des tissus. Selon DıjkshoorN, on assiste lors 
de l'absorption d'un cation à une compensation anionique simultanée sous forme de $\mathrm{OH}^{-}$ ou de $\mathrm{H} \mathrm{CO}_{3}{ }^{-}$, puis à la synthèse d'un anion organique. De même, l'absorption d'un anion minéral entraîne une compensation protonique avec décarboxylation d'un anion organique.

Il devenait donc nécessaire de compléter l'étude de la nutrition minérale de l'Épicéa par celle des anions organiques du tissu foliaire. Nous considérons toujours, rappelons-le, les acides organiques tantôt sous forme libre, tantôt sous forme salifiée.

Les teneurs en acides organiques des tissus prélevés au printemps et en fin de cycle de végétation sont très différentes selon qu'elles proviennent des stations calcaires ou décarbonatée. Cette différence est particulièrement marquée au stade printanier de croissance, lorsque l'activité biologique du tissu foliaire est la plus intense.

La production d'acides organiques n'est pas liée proportionnellement à la teneur en calcaire du sol; on observe en effet que, pour des taux de calcaire du sol aussi différentes que 15 p. 100 et 55 p. 100 , les teneurs en acides organiques présentes dans le végétal ne diffèrent pas significativement. L'acidité organique apparaît donc maximale pour une concentration au plus égale à 15 p. 100 de calcaire dans le sol. Il est difficile, compte tenu de l'aspect assez général de notre étude, de tirer des conclusions sur la signification physiologique de l'accroissement en acides organiques des tissus foliaires lorsqu'on passe d'un sol décarbonaté à un sol carbonaté. Nous pensons cependant que cet excès d'acidité organique observé sur sols calcaires reflète une métabolisation difficile de l'azote nitrique du sol, mais aussi probablement une synthèse protéique déficiente.

A l'opposé des acides organiques libres, leurs sels apparaissent sans relation avec le calcaire du sol; de plus, les teneurs observées ne dépendent que très peu de la période de prélèvement. D'une part l'indépendance des sels organiques vis-à-vis du milieu, d'autre part la faible variation de leur teneur pendant le cycle de végétation, apportent la preuve qu'ils peuvent être considérés comme un caractère propre à l'espèce étudiée. Des observations voisines ont été faites sur certaines plantes herbacées par DEwIT et Al (1963). Ces auteurs ont en effet mis en évidence dans les tissus l'existence d'une différence entre cations totaux et anions minéraux, caractéristique de l'espèce étudiée.

\section{2. - Répartition et nature des anions organiques (voir tableaux 3, 4, 5).}

Ces anions organiques peuvent être considérés comme dérivant d'acides libres et de leurs sels : acides aliphatiques, acides aminés, acides alicycliques, acides phénols. En ce qui nous concerne, nous n'avons répertorié que les anions essentiels. On peut en effet estimer d'après les travaux de Muir et Al (1964), de Bruckert (1970), que la participation des acides aminés et des acides phénols ainsi que de leurs sels est peu importante; ces anions représentent seulement 3 à 5 p. 100 de la totalité des anions organiques présents dans le tissu foliaire.

D'après les résultats obtenus, il apparaît que les anions organiques correspondant aux acides et aux sels d'acides organiques analysés peuvent être séparés en deux groupes : le premier comprend essentiellement les anions quinique et shikimique (anions alicycliques), le second les anions malique, succinique, citrique et oxalique.

L'anion quinique représente un peu moins de $1 / 3$ des anions organiques totaux présents dans le tissu foliaire de la station très carbonatée. Ce pourcentage est réduit de moitié sur station décarbonatée. Les contributions respectives de l'anion shikimique aux anions orga- 


\section{TABLEAU 4 - TABLE 4}

Identification des principaux anions organiques dans l'extrait soluble dans l'eau de tissu foliaire d'Épicéa réalisée sur des prélèvements hivernaux

Les résultats sont exprimés en mé/100 g de végétal sec. Ces anions sont représentés par la somme des anions organiques liés à un proton (acidité organique libre) et des anions organiques salifiés solubles dans l'eau.

Identification of principal organic anions soluble in water of Spruce sample during the winter season

The results are expressed in me/100 $\mathrm{g}$ of dry matter. These anions are composed of the organic anions fastened to $\mathrm{H}^{+}$(organic acids) and organic anions fastened to metals soluble in water.

\begin{tabular}{|c|c|c|c|c|c|c|c|}
\hline \multicolumn{3}{|c|}{ Stations } & \multicolumn{2}{|c|}{$\begin{array}{l}\text { Anions } \\
\text { totaux } \\
\text { solubles }\end{array}$} & \multicolumn{2}{|c|}{$\begin{array}{c}\text { Anions } \\
\text { minéraux } \\
\text { solubles }\end{array}$} & $\begin{array}{c}\text { Anions } \\
\text { organiques } \\
\text { solubles } \\
\text { (1) }\end{array}$ \\
\hline \multicolumn{3}{|c|}{$\begin{array}{l}\text { Station très carbonatée } \ldots \ldots \ldots \ldots \\
\text { Station décarbonatée } \ldots \ldots \ldots \ldots\end{array}$} & \multicolumn{2}{|c|}{$\begin{array}{l}64 \\
62\end{array}$} & \multicolumn{2}{|c|}{$\begin{array}{l}6 \\
9\end{array}$} & $\begin{array}{l}58 \\
53\end{array}$ \\
\hline \multicolumn{6}{|c|}{$\begin{array}{c}\text { Anions aliphatiques et alicycliques } \\
\text { solubles }\end{array}$} & \multirow{2}{*}{$\begin{array}{c}\text { Total } \\
(2)\end{array}$} & \multirow{2}{*}{$\begin{array}{l}\text { Indosés } \\
(1)-(2)\end{array}$} \\
\hline A.m* & A.s & A.c & A.q & A.sh & A. $\mathrm{O}_{\mathrm{x}}$ & & \\
\hline 4 & traces & 2 & 17 & 24 & traces & \multirow[t]{2}{*}{47} & 11 \\
\hline 4 & traces & 4 & 8 & 27 & 2 & & 8 \\
\hline
\end{tabular}

* Les abréviations sont celles du tableau 3.

N.B. - Les anions totaux solubles dans l'eau correspondent à l'acidité libre augmentée de l'acidité de déplacement des cations solubles par une résine échangeuse de protons.

\section{TABLEAU 5 - TABLE 5}

Teneur présumée en oxalate de Ca des tissus sur les prélèvements hivernaux

Les résultats sont exprimés en mé/100 $\mathrm{g}$ de végétal sec

Ca oxalat supposed content in foliar tissues on winter sample

Results are expressed in me/100 $\mathrm{g}$ of dry matter

\begin{tabular}{c|c|c}
\hline Stations & $\begin{array}{c}\text { Teneur en oxalate } \\
\text { de Ca* }\end{array}$ & $\begin{array}{c}\text { Teneur en anions } \\
\text { oxaliques insolubles** }\end{array}$ \\
\hline Station très carbonatée .... & 12 & 16 \\
Station décarbonatée ...... & 6 & 7 \\
\hline \hline
\end{tabular}

* Teneur en oxalates insolubles (oxalate de $\mathrm{Ca}$ ) obtenue par différence entre l'acidité organique totale (anions organiques totaux) et l'acidité organique soluble dans l'eau (anions organiques solubles dans l'eau). Ces analyses ont été conduites par titrimétrie à la soude.

** Teneur en anions oxaliques salifiés insolubles obtenue par différence entre les anions oxaliques totaux et les anions oxaliques sous forme d'acide et de sels solubles dans l'eau. Ces analyses ont été conduites par chromatographie sur papier. 
niques totaux sur station très carbonatée et décarbonatée sont de 34 p. 100 et 46 p. 100. Remarquons sur ces stations une contribution inverse des deux anions étudiés. Ceux-ci, de structure voisine, représentent environ 60 p. 100 de la totalité des anions organiques du tissu foliaire. Leur abondance se justifie dans la mesure où selon HARBORNE (1964), ils sont les précurseurs de la lignine et de nombreux composés aromatiques.

Les autres anions organiques se rencontrent à des teneurs faibles, quelle que soit la station étudiée; l'anion oxalique n'existe qu'à l'état de trace sous forme d'acide libre ou de sel soluble (tableau 4), dans les tissus foliaires de la station très carbonatée, alors que son existence est confirmée sous ces formes sur station décarbonatée.

Par contre, on observe des teneurs élevées, mais significativement différentes d'anions oxaliques combinés sous forme d'oxalate de $\mathrm{Ca}$ insoluble dans la station très carbonatée et décarbonatée. Celles-ci peuvent atteindre respectivement 28 et 15 p. 100 des anions organiques totaux.

\section{CONCLUSION}

\section{Nutrition et métabolisme du phosphore et du soufre}

En période d'activité biologique intense, il se produit dans le tissu foliaire provenant de stations très calcaires une accumulation des combinaisons minérales du phosphore soluble dans l'eau. Nous ne pensons pas que l'abondance de celles-ci soit directement la conséquence d'une absorption intense du phosphore minéral du sol, mais plutôt le résultat d'une transformation déficiente du phosphore minéral en phosphore organique dans le tissu foliaire. Il y aurait donc sur station très calcaire accumulation des formes solubles $\left(\mathrm{PO}_{4} \mathrm{H}_{2}{ }^{-}\right)$dans les tissus par défaut d'assimilation de cet anion.

Il y a identité de comportement entre les formes phosphorées et les formes soufrées. La présence, à des teneurs élevées, des combinaisons du soufre soluble dans l'eau dans les tissus foliaires de la station très carbonatée semble montrer, là aussi, qu'il pourrait s'agir d'une accumulation de soufre minéral résultant d'un défaut d'assimilation. Notons cependant qu'à l'inverse du phosphore, les différentes combinaisons du soufre analysées voient leurs teneurs s'accroître au cours de l'année de végétation.

\section{Nutrition azotée}

L'azote minéral sous forme $\mathrm{NO}_{3}{ }^{-}$et sous forme $\mathrm{NH}_{4}{ }^{+}$existe dans le tissu foliaire à des teneurs très faibles.

Un équilibre s'établit entre l'ensemble des formes azotées minérales, azotées solubles et insolubles du tissu foliaire, celui-ci ne dépend pas de la présence de calcaire dans le sol. Une des principales causes responsable de la carence en azote protéique observée sur sol calcaire serait liée à la prédominance de l'azote nitrique par rapport à l'azote ammoniacal sur ce type de sol. L'assimilation de cet anion étant vraisemblablement soumis à une réduction difficile et limitée du fait d'une faible activité de la nitrate réductase. 


\section{Relation entre les bilans nutritifs de l'azote et du soufre}

Nous avons retrouvé dans le tissu foliaire de Picea exselsa Link un rapport caractéristique soufre organique/azote organique indépendant du milieu stationnel de valeur voisine de celle trouvée par Kelly et LAmBert sur Pinus radiata. Si l'on se réfère aux travaux de Kelly et LAMBERT, la valeur constante de ce rapport tend à confirmer que les faibles teneurs en soufre organique insoluble relevées sur station très calcaire sont la conséquence directe de la carence azotée observée sur cette station.

\section{Nutrition cationique}

La totalité du $\mathrm{K}$ et la quasi-totalité du Mg se trouve dans le tissu foliaire à l'état soluble. Le calcium absorbé en quantité importante par l'Épicéa sur sol calcaire est rapidement fixé au niveau des tissus foliaires. L'interprétation de nos résultats montre que cette fixation de Ca peut s'effectuer suivant deux processus différents au moins.

On peut penser à la fois à une fixation par combinaisons avec des structures organiques (pectates de $\mathrm{Ca}$ ) se formant aussi bien sur sol calcaire que sur sol décarbonaté, ainsi qu'à une fixation sous forme de sels insolubles déplaçables par les résines plus précisément dans le cas d'un sol très carbonaté.

Incidence de la nutrition minérale sur la formation des acides organiques du tissu foliaire

Nous avons mis en évidence, aussi bien pour les tissus prélevés au printemps que pour les tissus prélevés en hiver, des teneurs en acides organiques différentes selon qu'ils proviennent des stations calcaires ou de la station décarbonatée. Ces acides sont représentatifs du type de nutrition azotée adoptée par le végétal.

Cette accumulation d'acides organiques rapportée à la matière sèche n'est pas proportionnelle à la teneur en calcaire. Elle est maximale dès la teneur de 15 p. 100 en calcaire du sol. A l'opposé des acides organiques libres, les acides organiques salifiés apparaissent sans liaison avec le calcaire. D'une part, leur indépendance vis-à-vis du milieu stationnel, d'autre part leur constance pendant la période de végétation apportent la preuve qu'ils peuvent être considérés comme caractère propre à l'espèce étudiée. L'analyse chromatographique a permis d'identifier les principaux acides organiques du tissu foliaire; parmi ces derniers les acides quinique et shikimique, précurseurs des lignines représentent ensemble environ 60 p. 100 de leur totalité.

Reçu pour publication en octobre 1974.

\section{REMERCIEMENTS}

Que soient remerciés ici tous ceux qui m'ont aidé dans la mise en œuvre de ce mémoire.

Je suis particulièrement reconnaissant à Monsieur MetCHE, Professeur de Biochimie à l'I.N.P. de NANCY, dont les idées et les critiques m'ont permis de mener à bien ces recherches. 


\title{
SUMMARY
}

\section{MINERAL NUTRITION OF Picea excelsa Link ON CALCAREOUS AND NON CALCAREOUS SOILS,} RESULT OF THE NUTRITION ON MINERAL AND ORGANIC ANIONS METABOLISM

This study has been realized on Picea excelsa Link from foliar samples in spring during the biological activity, and in winter during the biological pause. We have selected 3 soils : a very calcareous soil with 50 p. 100 of $\mathrm{CaCO}_{3}$, a calcareous soil with 15 p. 100 of $\mathrm{CaCo}_{3}$ and a non calcareous soil. Trees are 35 years old. On very calcareous soil results show a similar behaviour on the different forms of sulfur and phosphorus in foliar tissues. The low $\mathrm{S}$ and $\mathrm{P}$ insoluble contents show a difficult assimilation of the $\mathrm{S}$ and $\mathrm{P}$ soluble forms. For sulfur, assimilation is dependant of a deficiency of proteic synthesis. The different nitrogen combinations ratios in foliar tissues are not dependent of the calcareous in the soil. The principal reason of the low contents in proteic nitrogen observed on calcareous soil seems to be in relation with the mineral nitrogen's quality free in the soil.

We have noted an important absorption of $\mathrm{Ca}$.. on calcareous soil. The most part of this cation is insolubilised in the foliar tissues. These $\mathrm{Ca}$ insoluble combinations show that two process of insolubilisation would be possible. First $\mathrm{Ca}$ would be fixed as $\mathrm{Ca}$ organic compounds, non exchangeable with cationic resins, in all foliar tissues from every plot.

Then as $\mathrm{Ca}$ organic compounds exchangeable with cationic resins ( $\mathrm{Ca}$ oxalat). This organic compounds characterising especially the foliar tissues growing on the calcareous soil.

We have identified in the tissues the most important organic anions of the free organic acidity (organic acids) and the salt organic acidity (organic anions bounded to cations). The contents of free organic acids are higher in the foliar tissues growing on calcareous soils than on non calcareous.

\section{ZUSAMMENFASSUNG}

\author{
DIE MINERALERNÄHRUNG VON Picea excelsa Link AUF KALKHALTIGEN UND KALKFREIENBÖDEN, \\ EINFLUSS DER ERNÄHRUNG AUF DEN METABOLISMUS \\ DER ANORGANISCHEN UND ORGANISCHEN ANIONEN
}

Die vorliegende Untersuchung an der Fichte (Picea excelsa Link) ist durchgeführt worden, die, einerseits an Nadelproben im Frühjahr, als die biologische Tätigheit sehr stark war, und anderseits im Winter, während der biologische Ruheperiode, entnommen wurden. Drei Standorte wurden untersucht, ein sehr kalkhaltiger Boden mit $50 \%$ Kalk von der oberfläsche an, ein kalkhaltiger Boden mit $15 \%$ Kalk und ein kalkloser Boden. Die Bestände haben ein Alter von ungefähr 35 Jahren. Die Ergebnisse zeigen, auf sehr kalkhaltigen Böden ähnliche Anteile verschiedenen Schwefel und Phosphor verbindungen. Die gefundenen geringen Mengen unterstellt werden :

- Festlegung als unlöslicher organischer Komplex der durch den Harzkationen anstauscher nicht verlagert werden kann.

— Festlegung als Salz (unlösliches Oxalat) vor allem auf Kalkstandorten.

Die wesentlichen organischen Anionen d.b. die freien organischen Säuren und die durch den Austauscher freigemacht organischen Säuren wurden bestimmt. Es zeigte sich, dass der Gehalt an freien organischen Säuren in den Geweben auf kalkreichen Standorten höher ist als auf kalkarmen Böden.

Die gefundenen geringen Mengen von unlöslichen Schwefel und Phosphor dürften auf eine schlechte Assimilation der entsprechenden löslichen Formen zurückzuführen sein. Zumindest beim Schwefel scheint seine Aufnahme durch eine mangelhafte Proteinsynthese gegeben.

Zwischen den verschiedenen Stickstofformen im Blattgewebe (Mineralstickstoff, löslicher und unloslicher Stickstoff) stellt sich ein Gleichgewicht ein das vom Kalkgehalt des Bodens unabhängig ist. Eine der wesurtlichen Gründe für den auf Kalkstandorten beobachteten Mangel an Proteinstickstoff dürfte auf die Art des im Boden verfügbaren Mineralstickstoffes zurückzuführen sein. 
Der Ernährungszustand an Kationen ist auf kalkreichen Standorten insbesondere durch eine bedeutende Aufnahme von Kalzium gekenntzeichnet. Der grösste Teil dieses Ca ist unlöslich in den Geweben festgelegt, wofür zwei verschiedene Arten der Festlegung.

\section{RÉFÉRENCES BIBLIOGRAPHIQUES}

Barnes R.-L., Bengton G.-W., 1968. - Some aspects of nitrogen nutrition and metabolism in relation to fertilizer responses in southern pines. Forest Fertilisation Theory and Practice Published by the Tennessee Valley Authority.

BRUCKERT S., 1970. - Méthodes d'analyses des acides organiques et des composés phénoliques dans le sol. Centre de Pédologie biologique C.N.R.S. Nancy.

Document interne, 15 pages.

Clément A., 1970. - Analyses foliaires. Principes et protocoles analytiques. Document interne.

Clément A., 1972. - Étude expérimentale de l'échange cationique provenant de sels d'acides organiques solubles et insolubles réalisés sur Amberlite IR $120 \mathrm{H}^{+}$.

Application Extraction et dosage des acides organiques libres et salifiés dans les aiguilles de résineux. Document interne.

Clément A.,1972. - Étude de l'équilibre acido-basique de l'Épicéa (Picea excelsa Link) sur sol très carbonaté, carbonaté et décarbonaté. Thèse de spécialité - Université de Nancy.

Clément A., LeTACON F., 1975. - Observations sur le fonctionnement de la nitrate réductase et sur l'aptitude à réduire les nitrites par voie non enzymatique chez quelques résineux. Sous presse.

Coic Y., Lesaint C., Leroux F., 1961. - Comparaison de l'influence de la nutrition nitrique et ammoniacale combinée ou non avec une déficience en acide phosphorique, sur l'absorption et le métabolisme des anions-cations et plus particulièrement des acides organiques chez le maïs. Comparaison du maïs et de la tomate quant à l'effet de la nature de l'alimentation azotée. Ann. Physiol. vég. 3 (3), p. 141 à 163.

Dewit Ct., Dijkshoorn W., Noggle J.-C., 1963. - Ionic balance and Growth of plants. Versl Landbouwk Onderz $69-\mathbf{1 5}$, p. 1 à 70.

DiJkshoORN W., VAN WIJK A.-L. (1967) - The sulfur requierements of plants as evidence by the sulfur nitrogen ratio in the organic mater. A review of published data. Plant and Soil 26. 129-157.

Duchaufour Ph., Bonneau M., 1962. - Étude expérimentale de l'influence du calcaire sur la nutrition et la croissance d'un résineux acidiphile. Ann. de l'E.N.E.F. et de la Station de Recherches et Expériences, Tome XIX, Fasc. 3.

Harborne J.-B., 1964. - Biochemistry of Phenolics compounds. Academic Press. Berkely Square London W I.

Heller R., 1969. - Biologie végétale. Nutrition et métabolisme, Masson 1969, 578 p.

Hoffmann E., Fiedler J., 1966. - Die Stickstoffernahrung junger Koniferen. Biologische Rundschau 4 (3) S. 138-155.

Kelly J., Martia J., Lambert, 1972. - The relationship between sulphur and nitrogen in the foliage of Pinus radiata. Plant and soil. 37 395-407.

LETACON F., 1972. - Disponibilité de I'azote nitrique et ammoniacal dans certains sols de l'Est de la France. Influence sur la nutrition et la croissance de l'Épicea commun (Picea excelsa Link). Ann. Sci. forest., 1972, 30 (2) p. 183-205.

Letacon F., Millier C., 1970. - La nutrition minérale de l'épicéa commun (Picea excelsa Link) en sols carbonatés et en sols décarbonatés. Ann. Sci. forest., 27 (I), p. 63 à 88 .

Muir J.-W., Morrison R.-I., Bownc J., Logan J., 1964. - The Mobilisation of ion by aqueous extracts of plants. Composition of the amino acid and organic acid fractions of an aqueous extrat of pine needles. Journal of Soil Science vol. 15 N. 2. p. 220 à 225.

ТАмм C.-O., 1955. - Seasonal variation in the nutrient content of conifer needles - Medd. fr. skogsforskingsinstitut Band 45. no 5. p. 3-25.

VAN TuiL H.-D.-V. 1965. - Thesis Wageningen - Organic salts in plants in relation to nutrition and growth, 83 pages.

Weils G., Metz L.-J., 1963. - Variation in nutrient content of loblolly pine needles with season, age, soi and position on the crown Division S. 7. Forest and range soils. Soil. Sci. Soc. Amer 27. p. 90-93. 